\title{
Coarse Grades: Informing the Public by Withholding Information
}

\author{
Rick Harbaugh and Eric Rasmusen*
}

October 2012

\begin{abstract}
Certifiers of quality often report only coarse grades to the public despite having measured quality more finely, e.g., "Pass" or "Certified" instead of "73 out of 100". Why? We show that coarse grades result in more information being provided to the public because the coarseness encourages those of middling quality to apply for certification. Dropping exact grading in favor of the best coarse grading scheme always reduces public uncertainty because the extra participation outweighs the coarser reporting. In some circumstances, the coarsest meaningful grading scheme, pass-fail grading, is the most informative. JEL: D82, L15.
\end{abstract}

\footnotetext{
${ }^{*}$ Harbaugh: Kelley School of Business, Indiana University, Bloomington, IN (email: riharbau@indiana.edu). Rasmusen: Dan R. and Catherine M. Dalton Professor, Department of Business Economics and Public Policy, Kelley School of Business, Indiana University. Bloomington, IN (email: erasmuse@indiana.edu) We thank Mimi Chan, Mikkel Jakobsen, and Dan Zhao for research assistance, and Michael Baye and participants in talks at the BEPP Brown Bag Lunch, Baylor University, the Northeastern IO Conference and the Summer 2009 Econometric Society meeting for helpful comments.
} 


\section{Introduction}

Grades are often coarse. Rather than an exact number or rank, grades are usually only a rough indication of quality such as a letter grade or even just a pass-fail grade. For instance, many safety organizations certify that a product is safe with a seal of approval that does not indicate whether the product barely passed safety tests or passed them by a wide margin. And environmental organizations typically certify environmental quality with a simple "eco-label" based on a more detailed evaluation. They throw away information.

The loss of information from the coarsening of grades is often bemoaned, but why are grades coarse in the first place? An obvious reason is that it costs more to do fine grading than coarse grading. But this can't be the entire explanation since the certifier often collects detailed information but refrains from reporting most of it. Since certifiers deliberately reclassify information to be coarser before reporting the results publicly, coarse grading is at least as expensive as exact grading, not cheaper.

Many certifiers are non-profits with the explicit goal of providing the most information about products to consumers, making this coarsening even more of a puzzle. Most of the numerous eco-label schemes that provide information on products' environmental, health, and social impacts are run by non-profit organizations. This is seen from data provided by the website Ecolabelindex.com which tracks such certification schemes for a wide variety of products. Of the 363 different schemes tracked by the site, 209 are controlled by non-profits, 59 by industry groups, 53 by governments, and 42 by for-profit firms. ${ }^{1}$ Even when the certifier is trying to maximize profits, if its services are paid for by consumers or by advertisers, it has an incentive to provide accurate information so as to increase the value of its services.

When the certifier wants to provide accurate information to consumers, why make the information coarser than necessary? We suggest that the answer often lies in whether certification is voluntary. In many situations such as certification for eco-labels some costly cooperation from the firm is required so the certifier needs to consider the incentives of the firm to voluntarily participate. Just as a potential student is reluctant to go to a medical college that will publicly rank him as the worst student who graduated, a firm does not want to pay much for a seal of approval that tells the world it barely passed. Hence a certifier who wants to maximize information to consumers needs to

\footnotetext{
${ }^{1}$ We thank Anastasia O'Rourke for providing the data, which is from 2009.
} 
consider how the grading scheme affects the willingness of firms to be certified at all.

If the certification grade is coarse, mediocre firms are pooled with better firms so their expected quality conditional on the certification grade is higher than their true quality. Hence these firms have more incentive to bear the costs of certification and participation in the certification scheme rises. We show that, at the margin, the extra information from increased participation outweighs the loss in information due to coarseness, so the scheme that maximizes information to consumers paradoxically always involves some coarseness. Moreover, under plausible conditions the optimal scheme is maximally coarse: the product simply passes or fails, with no additional information provided.

In fact, we show that the optimal scheme is either pass-fail or a scheme with a combination of differentiation at the top and no differentiation among those who are good enough to be certified in equilibrium. The top firms are distinguished from each other and the lower firms are lumped together as certified, but while there is a theoretical failing grade, none of the firms that would receive it apply. Business Week ranks the top 30 MBA programs (of programs that express willingness to be ranked and provide all the necessary information) and then lumps remaining programs into a "second-tier" category. Recommendation letters work the same way: some students or employees won't even ask for a letter, some will receive favorable form letters, and the best will receive individuated letters.

We focus on the case where participation in a grading scheme is voluntary. Clearly the results are very different if participation is mandatory or if quality evaluation does not require the cooperation of the firm. For instance, camera companies cannot prevent online reviewers at Amazon.com or researchers at Consumer Reports from rating their products. In these cases there is no reason to hide information and indeed we usually see detailed ratings to help buyers, including explicit rankings of products. Similarly, governments agencies can often force firms to provide information about their products. In such cases, such as nutrition labels or energy usage labels, there is no need to encourage participation so we also expect to see requirements for provision of detailed information.

Our results help fill a significant gap in the literature. First, Lizzeri (1999) and others show how a certifier trying to maximize his own profits from charging for certification can do so by reporting product information coarsely. Second, Ostrovsky and Schwarz (2010), Gentzkow and Kamenica (2011) and others show how a certifier seeking to maximize perceived product quality (and hence the profits of firms being certified) can often do 
so by reporting information coarsely. Considering the three different parties involved in certification - certifiers, senders (firms), and receivers (consumers) - the existing literature assumes that the certifier acts to maximize his own benefits or the benefit to senders, while we assume that the certifier acts to maximize the information benefit to receivers. Our assumption would seem particularly unlikely to lead to coarse grading, but we find the same general result that the certifier injects some coarseness into his grading. We will say more about these and other papers, and discuss areas where our model makes different predictions from the literature, in the last section of this article.

\section{The Model}

A firm produces a product of quality type $q$ which is distributed on $[0,1]$ according to atomless distribution $F$ with density $f$ where $f>0$ on $(0,1)$. The realization of $q$ is the firm's private information, but a certifier can discover it if the firm submits the product for certification at cost $c$. The certifier first commits to a grading scheme, a mapping of measured quality $q$ to a message $m$. If the firm then applies for certification, the certifier measures its quality perfectly and reports message $m$ based on the grading scheme. If the firm chooses not to apply for certification, the certifier reports the message $m=$ "uncertified".

A consumer updates his estimate of quality $q$ based on the equilibrium meaning of $m$ and pays the firm this updated estimate, $E[q \mid m]$. The firm maximizes profit by the choice of whether or not to pay the cost $c>0$ for certification. The certifier is a nonprofit whose goal is to maximally inform consumers. ${ }^{2}$ We will define "maximally inform consumers" as to minimize the mean squared error (MSE) of the consumer's estimate of product quality. ${ }^{3}$

Our equilibrium concept is Perfect Bayesian Equilibrium, so for any grading scheme, consumer beliefs must be consistent with firm choices and follow Bayes Rule, and firm choices must be best responses to the consumer beliefs. If $c$ is so large that under a

\footnotetext{
${ }^{2}$ It does not matter whether there is just one certifier or many. Since the goal of a certifier is to maximize information to consumers, it will not engage in competition that worsens information, e. g. by providing exact grading to firms with very high quality to draw them away from (and destabilize) a pass-fail certifier.

${ }^{3}$ We have chosen MSE for concreteness, but the model can be adapted to other loss functions. If, for example, consumers care only that quality exceeds a threshold $x$, a pass-fail grading scheme does as well as any other scheme because the quality space is effectively binary too.
} 


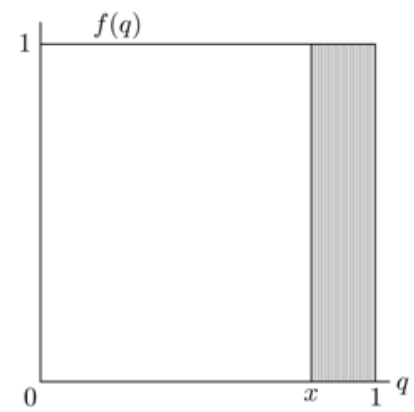

a) Exact Grading

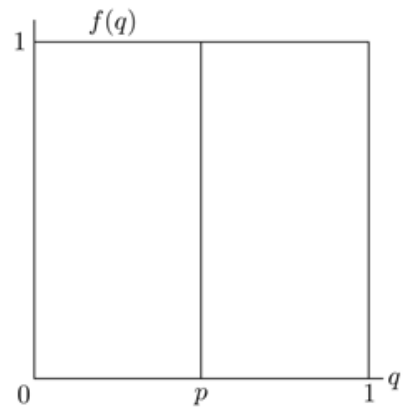

b) Pass-Fail Grading

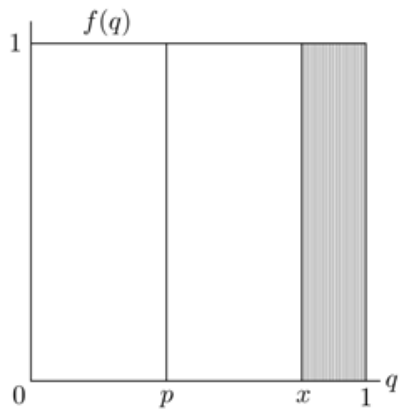

c) Honors Grading

Figure 1: Grading schemes for uniform distribution, $c=2 / 5$

grading scheme there exists no equilibrium in which any positive measure of firm types would apply for certification, we call that scheme infeasible. For any given scheme, we restrict attention to the most informative equilibrium. This restriction rules out equilibria where the consumer does not expect a firm to certify its quality and punishes unexpected certification with unfavorable off-equilibrium-path beliefs.

We allow for any arbitrary grading scheme, but there are three schemes of particular interest. In Figure 1(a)'s exact grading scheme the quality of the product is exactly revealed, with message $m=q$. There will exist some quality level $x$ such that all types $q \geq x$ have sufficient incentive to be certified, as we will explain below. Since those types are exactly revealed, the mean squared error of the public's estimate of $q$ under exact grading consists of the square of the error in estimating the quality of the uncertified firms in the quality interval $[0, x)$, or

$$
M S E_{\text {exact }}=\int_{0}^{x}(q-E[q \mid q<x])^{2} f(q) d q .
$$

If a scheme is not exact, it is coarse: the exact quality of at least some product types is not revealed. One of two kinds of coarse grading schemes will turn out to be optimal, depending on the circumstances. Figure 1(b)'s pass-fail grading is the coarsest grading scheme: the message is $m=$ "uncertified" if $q<p$ and $m=$ "pass" if $q \geq p \cdot{ }^{4}$ Assuming that $p$ is set so that all types $q \geq p$ have sufficient incentive to be certified, the mean

\footnotetext{
${ }^{4}$ Alternatively, the certifier could send the message "fail" but in equilibrium low-quality firms will not apply so a "fail" grade is off the equilibrium path. Farhi, Lerner, and Tirole (2011) show that revealing whether a firm tried to be certified but failed can be important when firms are uncertain of their own type, but that is not the case in our model.
} 
squared error of pass-fail grading is

$$
M S E_{\text {pass-fail }}=\int_{0}^{p}(q-E[q \mid q<p])^{2} f(q) d q+\int_{p}^{1}(q-E[q \mid q \geq p])^{2} f(q) d q .
$$

The other coarse grading scheme is Figure 1(c)'s honors grading, which sets a standard $x$ above which quality is revealed exactly but also divides types below $x$ into two groups by a passing standard $p$. If $q<p$ then $m=$ "uncertified", if $p \leq q<x$ then $m=$ "pass", and if $q \geq x$ then $m=q$. Assuming that $p$ and $x$ are set so that all types $q \geq p$ have sufficient incentive to be certified, the mean squared error of honors grading is

$$
M S E_{\text {honors }}=\int_{0}^{p}(q-E[q \mid q<p])^{2} f(q) d q+\int_{p}^{x}(q-E[q \mid q \in[p, x]])^{2} f(q) d q .
$$

If $p=x$ honors grading is equivalent to exact grading, and if $x=1$ it is equivalent to pass-fail grading, so we will require $p<x$ and $x<1$ for a scheme to be called "honors". Note that in each of these grading schemes the certifier does not report on a firm which fails to meet the certification standard so such firms are pooled with firms who do not apply.

\section{Why Coarseness Helps}

We will use examples based on specific quality densities to make three simple points. The first point uses the uniform density in Figure 2(a) to show how coarse grading can provide more information than exact grading by increasing participation. The second point uses the falling triangle density in Figure 2(b) to show how pass-fail grading can be not just better than exact grading but better than honors grading too. The third point uses the rising triangle density in Figure 2(c) to show how coarse grading can be feasible when exact grading is not. These are "can happen" results that illustrate the intuition, but in Section III we will generalize the results to broader classes of distributions.

\subsection{The Uniform Density}

First, suppose that the quality density $f$ is uniform as in Figure 2(a) and consider the exact grading scheme in Figure 1(a). The firm's payoff $(q-c)$ from certification is increasing in $q$, so some type $q=x$ has the least incentive to be certified. Since the payoff from not being certified is $E[q \mid q<x]=x / 2$, type $q=x$ is just indifferent between 


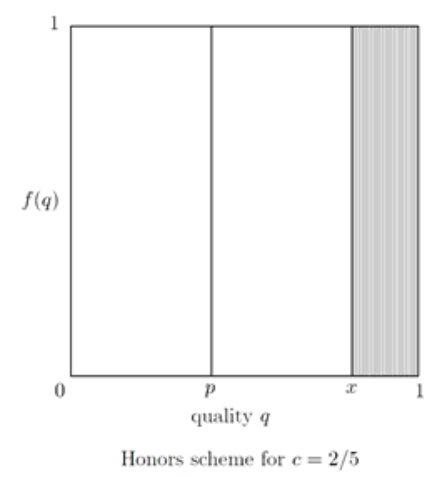

a) Uniform, $f(q)=1$

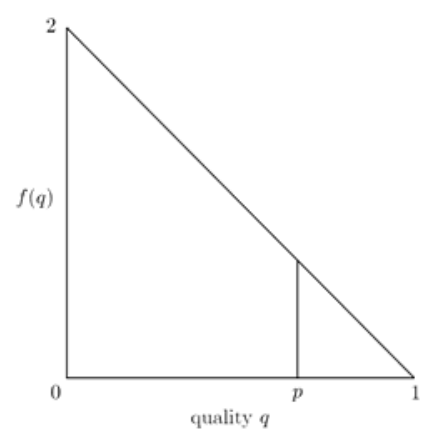

Pass-fail scheme for $c=1 / 2$

b) Downward Triangle, $f(q)=2-2 q$

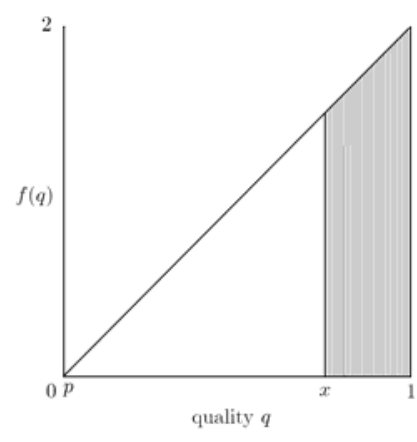

Honors scheme for $c=1 / 2$

c) Upward Triangle, $f(q)=2 q$

Figure 2: Distributions of product quality and optimal grading schemes

being certified and not if $x-c=x / 2$, so $x=2 c$. Thus, $M S E_{\text {exact }}=\int_{0}^{2 c}(q-c)^{2} d q=\frac{2}{3} c^{3}$ for the feasible range $c \leq 1 / 2$. As shown by Figure 3(a)'s "Exact" line, exact grading is perfectly informative as $c$ approaches 0 , since $x$ also approaches 0 . It is completely uninformative as $c$ approaches $1 / 2$, since $x$ approaches 1 .

Now consider Figure 1(b)'s pass-fail grading. For the uniform density, $E[q \mid q \in[p, 1]]-$ $E[q \mid q \in[0, p)]=(1+p) / 2-p / 2=1 / 2$, so any value of the cutoff $p$ is feasible as long as $c \leq 1 / 2$. The most informative cutoff under a symmetric convex loss function such as MSE is $p=1 / 2$, which from equation (2), has mean squared error of $M S E_{\text {pass-fail }}=$ $\int_{0}^{1 / 2}(q-1 / 4)^{2} d q+\int_{1 / 2}^{1}(q-3 / 4)^{2} d q=\frac{1}{48}$ in the feasible range. As seen from Figure 3(a)'s "P-F" line, pass-fail grading provides more information to consumers than exact grading when $c$ is large enough that few types will be certified under exact grading. Although pass-fail grading provides only noisy information, more middling types are willing to be certified since they can pool with high types, and the extra information on these types more than compensates for the extra noise.

Pass-fail grading does better than exact grading for high $c$, but honors does even better. Figure $1(\mathrm{c})$ 's honors grading cuts region $[0, x)$ in two using the passing standard $p$. Suppose we leave $x=2 c$ as before and set $p=c$, so the lower region is divided evenly. Types in the exact region $q \geq x$ now have even more incentive to be certified rather than look like a bad type who cannot even pass. Types in the new pass region gain $E[q \mid q \in[p, x)]-E[q \mid q \in[0, p)]=(p+x) / 2-p / 2=x / 2$ from passing so at $x=2 c$ this gain just covers the certification cost. Therefore all types $q \geq p=c$ will participate and $M S E_{\text {honors }}=\int_{0}^{c}(q-c / 2)^{2} d q+\int_{c}^{2 c}(q-3 c / 2)^{2} d q=\frac{1}{6} c^{3}$ as shown 


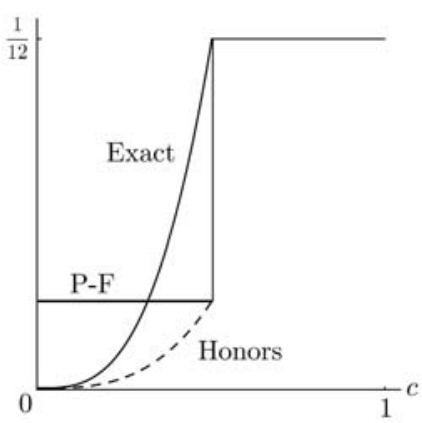

a) $f(q)=1$

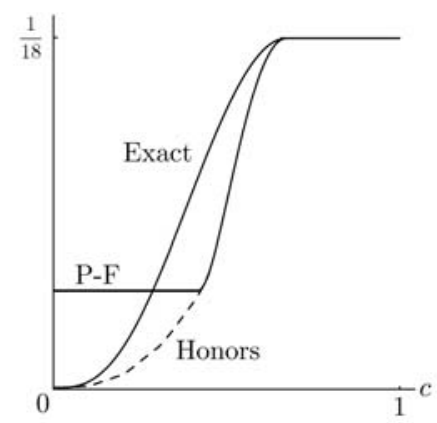

b) $f(q)=2-2 q$

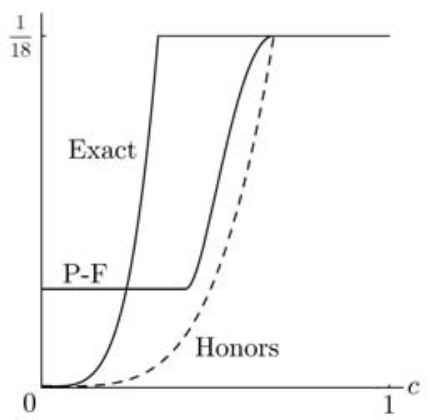

c) $f(q)=2 q$

Figure 3: MSE for different schemes and different distributions

by Figure 3(a)'s "Honors" line. By allowing for more participation and continuing to provide exact information on high types, honors grading outperforms both exact and pass-fail grading.

Our first result, which will be shown to hold generally in Proposition 1, follows from the above discussion.

Remark 1 Coarse grading can increase information by increasing participation.

\subsection{The Falling Triangle Density}

To see how pass-fail grading can outperform honors grading, consider the falling triangle density $f=2-2 q$ in Figure 2(b), which has the property that the gain from passing, $E[q \mid q \in[p, x)]-E[q \mid q \in[0, p)]=\frac{2}{3}\left(\frac{x}{2-p}\right)$, increases in $p$. Consider pass-fail grading so $x=1$ and first suppose that $c$ is low, which we will see makes honors grading the best. When the certifying cost $c$ is low, $p$ can be set to divide the region $[0,1]$ to minimize MSE without the participation constraint being binding. In particular, from minimization of (2), the best division is at $p=\frac{3}{2}-\frac{1}{2} \sqrt{5} \approx 0.382$, which is feasible for $c<\frac{1}{3} \sqrt{5}-\frac{1}{3} \approx$ 0.412 . Within the range $[0, .412]$ there is slack in the pass-fail participation constraint for those types that apply; the types near $q=1$ can be exactly revealed and $p \approx .382$ is still optimal and feasible. Thus, as with the uniform density, the certifier can do even better by using honors grading. This is seen in Figure 3(b) where for low $c$, honors grading reduces MSE relative to pass-fail grading.

As $c$ rises the participation constraint becomes binding. The gain from passing is increasing in the cutoff $p$, so $p$ will have to be set higher to ensure participation. If $x$ 
falls from $x=1$ to $x^{\prime}<1$, to have honors grading the gain from passing must fall, so $p$ will have to be set even higher to ensure participation, to some value $p^{\prime}>p$. Honors grading is no longer "for free," as with the uniform density; it comes at a tradeoff. It provides more information on types $q \in\left[x^{\prime}, 1\right]$, who are exactly revealed, and on types $q \in\left[p^{\prime}, x^{\prime}\right)$, who are in a smaller pooling group, but less information on uncertified types $q \in\left[0, p^{\prime}\right)$, who form a larger pooling group. If $c$ is such that the uncertified group is already sufficiently large, the information loss from additional noise about the group dominates and MSE rises. Figure $3(\mathrm{~b})$ shows this. For $c \gtrsim .412$, the optimal honors grading scheme has $x=1$, which is equivalent to pass-fail grading.

Our second result, which is generalized and extended in Proposition 2, follows.

Remark 2 The coarsest of the three grading schemes - pass-fail grading - can be the most informative.

\subsection{The Rising Triangle Density}

So far we have concentrated on how coarse grades can increase participation by more types and thereby increase informativeness. We now focus on feasibility: when can a scheme induce any participation at all? For the uniform density the schemes induce different amounts of participation, but all of them can induce at least some participation for $c<1 / 2$ as seen in Figure 3(a). Similarly, for the falling triangle density each scheme is feasible for $c<2 / 3$, as seen in Figure 3(b).

As an example of how coarse grading can be feasible when exact grading is not, consider the rising triangle density $f(q)=2 q$ in Figures 2(c) and 3(c). Under exact grading, the gap $x-E[q \mid q<x]=x-2 x / 3=x / 3$ reaches a maximum of $1 / 3$ at $x=1$, so exact grading is feasible for $c \leq 1 / 3$. Under pass-fail grading, the gap

$E[q \mid q \geq p]-E[q \mid q<p]=\frac{2}{3}\left(\frac{1}{1+p}\right)$ is decreasing in $p$ and converges to a maximum of $2 / 3$ as $p$ approaches 0 . Therefore, pass-fail grading is feasible for $c \leq 2 / 3$. Since honors grading can use an $x$ arbitrarily close to 1 , honors grading is also feasible for $c<2 / 3$.

Our third result, which is generalized and extended in Proposition 3, follows.

Remark 3 Coarse grading can be feasible when exact grading is not.

These examples show the advantages of coarse grading and in particular show that the shape of distributions can have a large impact on the informativeness and feasibility of different grading schemes. 


\section{Propositions for General Distributions of Quality}

The above analysis compared the informativeness and feasibility of the exact, pass-fail, and honors grading schemes for particular distributions. We now generalize our analysis to include any grading scheme and any quality density $f$ that has full support and no atoms.

The following properties of the conditional means of a distribution for regions above and below a cutoff are central to our analysis as we saw for particular examples in the previous section, and as we will see more generally in this section.

CONDITIONAL MEANS PROPERTIES For any continuous density $f(q)$ with full support on an interval and any $t$ in that interval:

(i) For any $f, \frac{d}{d t} E[q \mid q>t]>0$ and $\frac{d}{d t} E[q \mid q<t]>0$.

(ii) For strictly logconcave $f$ (or $F), \frac{d}{d t} E[q \mid q>t]<1$, and $\frac{d}{d t} E[q \mid q<t]<1$.

(iii) For uniform $f$ (linear $F), \frac{d}{d t}(E[q \mid q>t]-E[q \mid q<t])=0$.

(iv) For strictly decreasing $f$ (strictly concave $F), \frac{d}{d t}(E[q \mid q>t]-E[q \mid q<t])>0$.

(v) For strictly increasing $f$ (strictly convex $F), \frac{d}{d t}(E[q \mid q>t]-E[q \mid q<t])<0$.

PROOF: Properties (i-iii) are standard while properties (iv) and (v) are provided by Jewitt (2004) as implications of more abstract general results. We provide direct proofs of properties (iv) and (v) in the Appendix.

Property (i) states that as the cutoff $t$ rises the means of both the lower region and the higher region rise. This follows since some types who are below average in the upper region are shifted into the lower region where they are above average. Property (ii) implies that for a log-concave distribution these means do not rise too rapidly. This property is useful for understanding exact grading. In particular, the second part tells us that if there is a cutoff $t$ such that all types above that cutoff are exactly revealed $(x=t)$, then raising $t$ increases the mean of the uncertified region at a rate slower than 1. As a result, the gap $t-E[q \mid q<t]$ between the marginal type who is graded exactly and the average quality of the uncertified pool is increasing in $t$. This gap is the maximum amount that the marginal type is willing to pay to be certified, so when $f$ is logconcave the cutoff for the most informative feasible exact grading scheme increases 
with certification costs. Most standard densities (see Bagnoli and Bergstrom, 2005) and all of our specific examples are logconcave, so finding the most informative exact grading scheme is straightforward.

Properties (iii-v) say how the gap between the upper and lower means change with $t$. For $p=t$ this gap is the most a type in the pass region is willing to pay to be separated from the uncertified pool. Property (ii) says that for uniform $f$ the gap is constant in $t$, property (iii) that for decreasing $f$ the gap falls with $t$, and property (iv) that for increasing $f$ the gap rises with $t$. A decreasing density puts relatively more mass at the lower end of each region, so the impact of a $t$ shift on the means is bigger for the upper region and the gap in means rises. Similarly, an increasing density's impact on the mean is smaller for the upper region and thus a $t$ shift reduces the gap. ${ }^{5}$ As we saw in the examples of the previous section, these differences determine how high or low a $p$ is feasible for any given cost of certification.

For our first general result we extend Result 1, which showed that coarse grading can outperform exact grading using the uniform distribution as an example. Recall that for the uniform distribution, turning exact grading into honors grading by introducing a "pass" region is always possible. We start with the exact scheme $x^{*}$ and introduce honors grading by setting $p=p^{\prime}$ slightly below $x^{*}$. Will types in $\left[p^{\prime}, x^{*}\right)$ still participate? Yes, for uniform $f$ because the gain from passing relative to failing, $E\left[q \mid q \in\left[p, x^{*}\right)\right]-E[q \mid q \in$ $[0, p)]=x^{*} / 2$ is constant in $p$ for a given $x^{*}$. For the rising triangle distribution and more generally for any rising $f$ as shown in Property (v), $E\left[q \mid q \in\left[p, x^{*}\right)\right]-E[q \mid q \in[0, p)]$ is decreasing in $p$ so again it is always possible to introduce a pass region with $p^{\prime}<x^{*}$ that does not affect the proportion of types who are exactly grade. But more generally, if the decrease to $p^{\prime}<x^{*}$ reduces the pass region's mean more than the fail region's mean then the participation constraint is no longer met for $x=x^{*}$. Hence $x$ will have to rise in order to maintain the incentive to participate, but this causes a loss in information so it is no longer clear whether there is a net benefit from introducing the pass region. This tradeoff happened with the falling triangle distribution and Property (iv) tells us that it arises for all decreasing $f$.

Figure 4(a) shows a (logconcave) distribution with a large region of decreasing $f$ so that one might expect the same tradeoff as well. ${ }^{6}$ Indeed for this distribution and with

\footnotetext{
${ }^{5}$ These results on the differences in means have broad implications for signaling games with binary signals and are used most notably in Benabou and Tirole (2005).

${ }^{6}$ This is the Beta $(2,3)$ distribution, $f(q)=12 q(1-q)^{2}$. Note that the uniform distribution is $\operatorname{Beta}(1,1)$, the falling triangle distribution is $\operatorname{Beta}(1,2)$, and the rising triangle distribution is Beta $(2,1)$.
} 


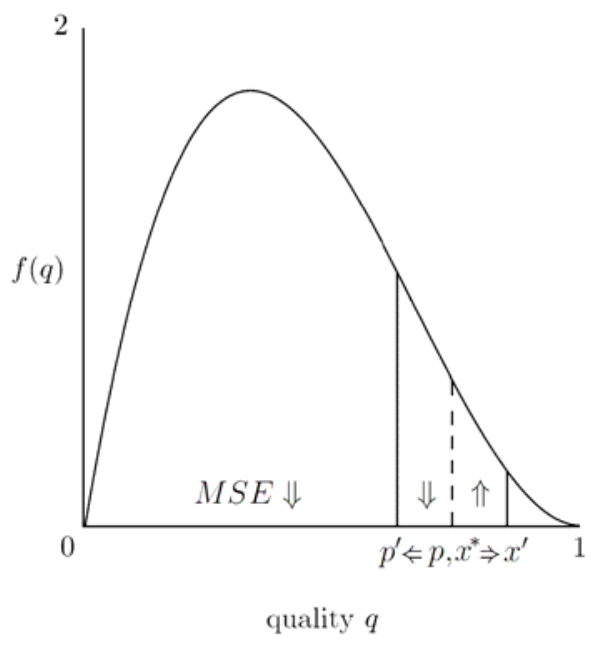

a) From Exact to Honors Grading

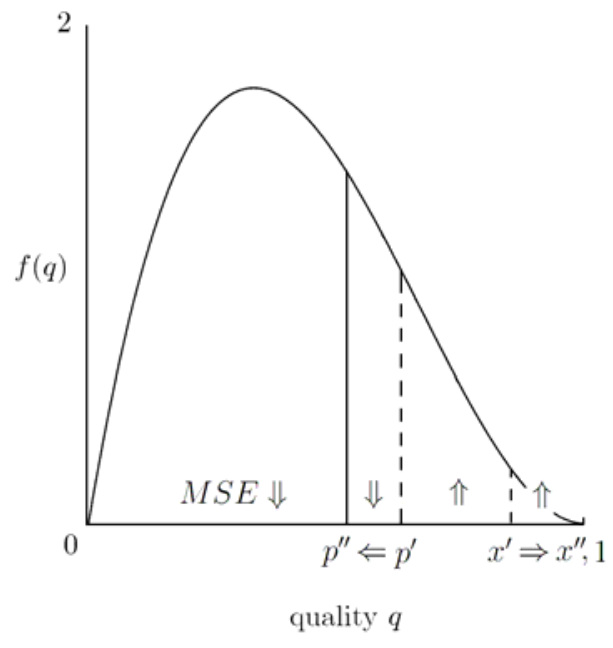

b) From Honors to Pass-Fail Grading

Figure 4: Effect of coarseness on MSE

costs $c=2 / 5$ introducing a pass region with $p^{\prime}<x^{*}$ requires increasing the exact cutoff from $x^{*}$ to some $x^{\prime}>x^{*}$ so as to maintain participation. This change splits the quality types into four groups. The first group, $\left[0, p^{\prime}\right)$, contributes less to MSE than under exact grading, because it is a smaller pool, with its types closer to its mean. The second group, $\left[p^{\prime}, x^{*}\right)$, also contributes less, because those types were pooled into the fail interval before, and the fail interval included more diverse types than the new pass interval (since the interval $\left(x^{\prime}-p^{\prime}\right)$ is not too large). The third group, $\left[x^{*}, x^{\prime}\right]$ is where the contribution to MSE increases. Under exact grading, those types were exactly revealed, but now they are pooled in the pass interval. The fourth group, $\left[x^{\prime}, 1\right]$, has no effect on MSE because it is exactly revealed under both exact and honors grading. So the question is whether the information gains for the first and second groups exceed the information loss for the third group.

The following proposition shows that, despite the possibility of loss from putting some formerly revealed firms into a pool, it is always possible to find a coarse scheme that outperforms the best exact grading scheme. The idea is that for marginal decreases in $p^{\prime}$ below $x^{*}$ there is a first order effect on the first group but only a second order effect on the third group (and also second group) because the pooling pass interval $\left(x^{\prime}-p^{\prime}\right)$ is so small. The result holds for the example in Figure 4(a) and more generally. Moreover,

The Beta distribution is logconcave for all parameter values. 
of all possible grading schemes - not just the three we have highlighted - the best will be either pass-fail or honors.

Proposition 1 For any quality density $f$, the most informative feasible grading scheme is either pass-fail or honors.

PROOF: See the Appendix.

This result shows that either pass-fail or honors grading is superior to exact grading or more complicated schemes, but does not say when honors grading is superior to passfail and vice-versa. ${ }^{7}$ The following proposition deals with this question. For small $c$ we find that honors grading outperforms pass-fail grading, as was seen in all of the examples in Figure 3. We find the opposite result for large $c$ and decreasing $f$ as in Figures 2(b) and $3(\mathrm{~b})$. In this case pass-fail grading outperforms honors grading.

Proposition 2 i) For c sufficiently low and any quality density $f$, honors grading is most informative, exact grading is next, and pass-fail is least informative:

$$
M S E_{\text {honors }}<M S E_{\text {exact }}<M S E_{\text {pass-fail }}
$$

ii) For c sufficiently high and $f$ decreasing, if any scheme is feasible then pass-fail grading is most informative, honors is next, and exact grading is least informative:

$$
M S E_{\text {pass-fail }}<M S E_{\text {honors }}<M S E_{\text {exact }} .
$$

PROOF: See the Appendix.

To better understand why pass-fail grading can do better for high certification costs, look at Figure 4(b). This is the same case as Figure 4(a) except that the pass region has been expanded so much that the honors region has been eliminated. If we start with the honors scheme $\left(p^{\prime}, x^{\prime}\right)$ in Figure $4(\mathrm{a})$ and increase the pass region by dropping $p^{\prime}$ to $p^{\prime \prime}$, we must also increase $x^{\prime}$ to maintain feasibility. Since costs are high and we started towards the upper end of the density, as we increase the pass region enough, the honors region shrinks to zero, so we have a pure pass-fail scheme. Types in the interval $\left[0, p^{\prime \prime}\right)$ contribute less to total MSE since the pool is tightened so each type is closer

\footnotetext{
${ }^{7}$ Recall that we require $x<1$ for "honors grading" so pass-fail grading is not merely a subset of honors.
} 
to the conditional mean. Types in $\left[p^{\prime \prime}, p^{\prime}\right)$ also contribute less since the types in this region have been moved from a larger to a smaller pool. However the opposite is true for types in $\left[p^{\prime}, x^{\prime}\right)$ because the pool these types are in has expanded, and also for types in region $\left[x^{\prime}, 1\right]$ since these types were formerly exactly revealed. Overall we have gained information (lowered MSE) on types in $\left[0, p^{\prime}\right)$ and lost information (raised MSE) on types in $\left[p^{\prime}, 1\right]$. The proof of Proposition 2 shows that for $f$ everywhere decreasing this always adds up to a net improvement in information for $c$ sufficiently large. Numerically for the case in the figure with a large decreasing region we find that for $c=2 / 5$ or greater $x=1$ in the optimal scheme so pass-fail grading is best.

Sometimes exact grading is not only less informative than coarse grading but also infeasible. A confused certifier who tried to use exact grading despite its inferiority would find that nobody at all would show up to be graded. This was the case in Figure 3 (c) (the rising triangle density) for exact grading even for many values of $c$ for which pass-fail and honors remained feasible. Proposition 3 gives more general conditions for when exact grading is inferior in feasibility to other grading schemes.

Proposition 3 (i) For any quality density $f$, if exact grading is feasible then so is honors grading, and if honors grading is feasible then so is pass-fail. (ii) For $f$ increasing, or for $f$ log-concave and $E[q]>1 / 2$, pass-fail and honors grading are feasible for a range of grading costs so high that exact grading is not.

PROOF: See the Appendix.

\section{Extension: Letter Grading with Different Con- sumer Prior Information on Different Firms}

So far we have followed the standard assumption in the literature of a single firm drawn from a distribution or, equivalently, multiple firms from the same distribution. Now suppose the certifier must use the same grading scheme for multiple firms when it is common knowledge that firms have different quality distributions. For instance, for environmental quality it might be known by consumers that one firm is better than another, but only the firm knows for sure.

We are particularly interested in when tiered certification in the form of "letter grades" is optimal. For instance the LEED certification system for building environmental impact has "silver", "gold", and "platinum" categories. For a firm with a good 


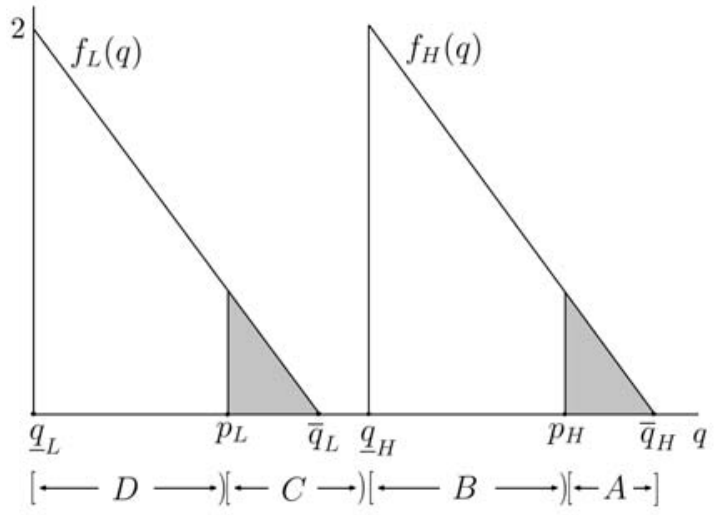

a) Disjoint distributions

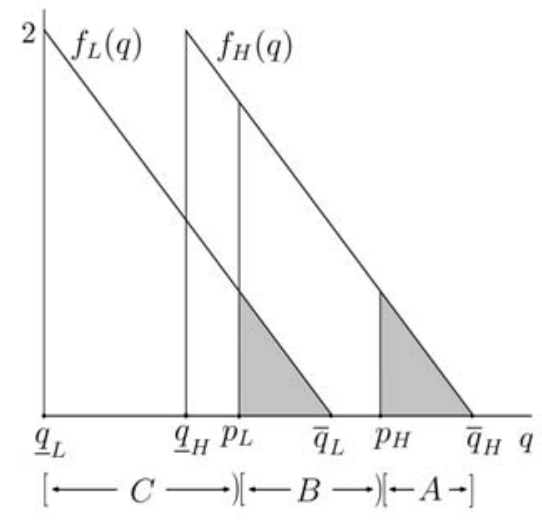

b) Overlapping distributions

Figure 5: Letter grading with different priors

reputation, receiving just a "silver" rating might not be worth the certification cost, but for a firm with a bad reputation such a rating might well be worth it. Hence, having different tiers might increase participation when consumers have different prior distributions about different firms.

Consider a setting in which firm qualities follow downward triangle densities and consumers know whether a firm is drawn from the low-tier density over $\left[\underline{q}_{L}, \bar{q}_{L}\right]$ or the high-tier over $\left[\underline{q}_{H}, \bar{q}_{H}\right]$. If the two types of firms don't overlap $\left(\bar{q}_{L}<\underline{q}_{H}\right)$, the optimum for sufficiently high costs is from our example demonstrating Remark 2, pass-fail for each tier of firm. Let this optimal pass-fail cutoff be $p_{L}$ for the low tier and a $p_{H}$ for the high tier. ${ }^{8}$ As shown in Figure 5(a) we could reframe this as a system with four grades, $\mathrm{C}$ and $\mathrm{D}$ for the low tier and $\mathrm{A}$ and $\mathrm{B}$ for the high tier.

In the example, A's and C's would be awarded as shown by the shaded areas of the figure, but there would be a "hole" with no B's observed since types would not want to pay $c$ to be certified as being in the bottom region of their tier. Could there be an equilibrium with no such holes? Yes, if we modify the example slightly to the overlapping distributions in Figure 5(b). Let the two densities overlap moderately with $\underline{q}_{H}<p_{L}<\bar{q}_{L}<p_{H}<\bar{q}_{H}$, keeping the same relative values for the cutoffs $p_{L}-\underline{q}_{L}$ and $p_{H}-\underline{q}_{H}$ that were optimal when there was no overlap. The following is a Perfect Bayesian Equilibrium:

\footnotetext{
${ }^{8}$ Similar results would follow from densities that first rise and then fall if the rising portion is small enough and $c$ is large enough.
} 
The certifier assigns $C$ 's for $\left[0, p_{L}\right), B$ 's for $\left[p_{L}, p_{H}\right)$, and $A$ 's for $\left[p_{H}, \bar{q}_{H}\right)$. High-tier firms in $\left[p_{H}, \bar{q}_{H}\right]$ apply and get $A$ 's. Low-tier firms in $\left[p_{L}, \bar{q}_{L}\right]$ apply and get B's. The remaining firms do not apply.

The firms which apply cannot do better by being uncertified, because the cutoffs were chosen in the original example to make this unprofitable and nothing has changed in the firms' incentives . Even if consumers had the peculiar out-of-equilibrium belief that the quality of a firm that deviated by not applying was near the cutoff rather than at the mean of firms that in equilibrium do not apply, the deviation would be unprofitable. The low-tier firms that do not apply already have the same expected value as a $\mathrm{C}$ would give them. The high-tier firms in $\left[p_{L}, p_{H}\right]$ that do not apply would receive B's if they applied, but the cost $c$ is too high for that to benefit them. Given the cost $c$ and the cutoff $p_{H}$, a high-tier firm in $q=\left[p_{H}, \bar{q}_{H}\right)$ would be indifferent between (i) paying $c$ and being labelled as an A (whatever its true value was), and (ii) remaining uncertified. ${ }^{9}$

Is the grading scheme still optimal for the certifier? Yes, since we assume the overlap between low-tier and high is small enough. A slight reduction in $p_{H}$ would divide the lowtier types slightly better, but it would cause all the high-tier types to drop certification, because the average quality of the pool $\left[p_{H}, \bar{q}_{H}\right)$ of high-tier firms would become too high. A big reduction would not be worthwhile because the small overlap means there are only a few low-tier firms with $\left[q=\underline{q}_{H}, p_{H}\right)$, the group about which information would improve. A slight increase in $p_{L}$ would have no effect at all except to worsen information on the low tier. A large enough increase would bring $q_{L}$ above $\underline{q}_{H}$ so possibly some hightier firms would now apply and receive B's, but the number of low-tier types receiving C's would fall more (since the overlap region is small) than the number of high-tier types receiving B's would rise. Thus, a small amount of overlap between the tiers would not cause the certifier to change his grading scheme.

We have therefore seen that when consumers have independent information about firm quality, the combination of that information with certifier grading can lead to more complicated grading schemes being optimal, including those with multiple grades. The reasoning of Proposition 1 still applies, however. The optimal scheme must be coarse because, at the margin, introducing a pass-fail cutoff right below the cutoff for an exact scheme always reveals more information about previously uncertified types than it hides about already certified types. This logic combined with the examples gives us

\footnotetext{
${ }^{9} \mathrm{~A}$ high-quality firm in $q=\left(\underline{q}_{H}, p_{H}\right)$ would strictly desire to remain uncertified, which is why all the observed B's will be low-tier firms.
} 
the following proposition.

Proposition 4 If the same grading scheme is used for different firms with different distributions, the most informative scheme is always coarse and sometimes uses only letter grades with no interval exactly revealed.

\section{Discussion and Connection to the Literature}

We have shown that a certifier who is trying to maximize information to the public should, paradoxically coarsen his information before reporting it. Rather than simply revealing what he has measured, the certifier will reveal only part of what he knows, and in some situations will only reveal whether a firm passes a quality threshold. The certifier faces a tradeoff between coarse grading, which attracts more firms to be certified, and fine grading, which informs the public better about the firms attracted. We show that the optimal tradeoff always involves some coarseness.

Two strands of the literature are most related to the situation we model. The first looks at the alternative setting in which a for-profit certification intermediary designs a scheme to maximize rent extraction from firms afraid of consumer beliefs about their quality if they lack certification. Lizzeri (1999) shows that the profit-maximizing scheme is a pass-fail disclosure where all but the very worst type of firm receives a passing grade. By certifying almost everyone, the certifier can extract a certification fee from almost

everyone. And since each firm is afraid of being pooled with the minute number of bottom-quality firms who remain uncertified, the fee can be large. Our model differs from this paper and those that follow it in assuming that the certifier aims to maximize consumer information, as in the case of a non-profit certifier who charges just enough to cover the costs of certification or who charges nothing but requires firms to bear some cost in providing information. The certifier introduces coarseness not to gain profits but to induce participation by firms who do not want their exact quality revealed.

A second strand of the literature concerns certifiers whose goal is to maximize the benefit to the subjects being certified. That literature introduces strategic reasons to obscure the information the public receives - indeed, the ideal would be to perfectly fool the public. Ostrovsky and Schwarz (2010) consider colleges which choose grading schemes to maximize the success of their students on the job market. Depending on the distributions of jobs and student abilities, students overall may gain if weaker students are pooled together with better students. The average quality of students at Harvard 
might be so high that they will all get jobs if their grades are so uniformly high as to be uninformative, but weaker students might not get jobs if they are revealed as weak. In their model, students are exogenously assigned to colleges, so colleges are free to ignore the selection effect we study in the present paper. Similarly, Chakraborty and Harbaugh (2007) consider the gains from coarse rankings of students in a cheap talk model. Rayo and Segal (2010) and Kamenica and Gentzkow (2011) consider the general problem of the sender itself - the firm or student - committing to a disclosure policy, rather than a certification intermediary doing so, and find that a coarse disclosure policy often benefits the sender. Our model differs from these because the certifier's objective is to inform the public, not to mislead it and because firms can choose not to be certified. Coarseness in these models reduces information to the public without any gains from encouraging participation.

Other important reasons for coarse grading have also been analyzed in the literature but we have abstracted from them in our model. We assume that coarse and fine grading are equally costly, but Titman \& Trueman (1986), Farhi, Lerner \& Tirole (2011), and others consider the case where finer grading costs more. We assume that the certifier can provide verifiable information about quality, but it might be that the certifier's credibility is not assured, in which case a coarse report can be more credible than an exact report (Crawford \& Sobel, 1982; Morgan \& Stocken, 2003). Finally, we take quality as exogenously given but certification plays a role in giving firms an incentive to expend effort to improve quality. If the certifier is trying to maximize the amount of effort by those being certified, coarse grades can induce more competition when abilities are heterogeneous (Dubey \& Geanakoplos, 2006; Moldovanu, Sela \& Shi, 2007). ${ }^{10}$

Our approach adds to this rich literature by showing how coarseness is optimal in the environment that would seem least conducive to it - when the certifier is explicitly trying to maximize information to the public and there are no difficulties in providing exact grades. Since coarseness arises from the incentive to encourage participation, a distinctive prediction of our model is that certification should be coarse or exact depending on whether certification is voluntary or mandatory. At Ecolabelling.org, a website that collects information on labelling, we found that of the 174 voluntary labels from OECD countries for which grading data could be found, only 5 of them provide exact grades or grades with more than a few levels. ${ }^{11}$ In contrast, all 5 of such

\footnotetext{
${ }^{10}$ Costrell (1994) considers the related question of how to set a pass-fail standard to maximize student effort.

${ }^{11}$ Many of them provide additional separate recognition of exceptional firms, though not necessarily
} 
mandatory labels provide fine or exact grades. The voluntary US Energy Star label for home products only indicates that the product has met a certain standard for low energy usage, but the EnergyGuide label that is mandatory for large appliances provides exact information on energy usage and expected energy cost.

Based on the idea that private sector labels might be responding to consumer demand for simplicity in providing labels with only coarse information, there have been proposals for government agencies to also adopt labels with less precise information. For instance, Congress in 2005 directed the FTC to consider switching the mandatory EnergyGuide label to a coarse star-ranking scheme. ${ }^{12}$ From the perspective of our model, where coarseness is driven by firm participation incentives rather than consumer difficulty in comprehending exact labels, such coarseness only makes sense when government certification schemes are voluntary. For mandatory schemes where participation incentives are not a factor, our model implies that shifting away from exact grades would hurt rather than help consumers.

\section{Appendix}

CONDITIONAL MEANS PROPERTIES (iv) For strictly decreasing $f, \frac{d}{d t}(E[q \mid q>$ $t]-E[q \mid q<t])<0$. (v) For strictly increasing $f, \frac{d}{d t}(E[q \mid q>t]-E[q \mid q<t])>0$.

PROOF: Integrating by parts, note that for $a \leq b$,

$$
E[q \mid q \in[a, b]]=\frac{\int_{a}^{b} f(q) q d q}{F(b)-F(a)}=\frac{b F(b)-a F(a)}{F(b)-F(a)}-\frac{\int_{a}^{b} F(q) d q}{F(b)-F(a)} .
$$

(iv) First suppose $f^{\prime}<0$. We will show that $\frac{d}{d t} E[q \mid q>t] \geq 1 / 2$ with possible equality only at $t=1$, and that $\frac{d}{d t} E[q \mid q<t] \leq 1 / 2$ with possible equality only at $t=0$. From $(4),{ }^{13}$

$$
\frac{d}{d t} E[q \mid q<t]=\frac{d}{d t}\left(t-\frac{\int_{0}^{t} F(q) d q}{F(t)}\right)=\frac{\int_{0}^{t} F(q) d q f(t)}{F(t) F(t)}
$$

on the label itself.

${ }^{12}$ Ultimately it was determined that consumers learned most from exact information about expected energy costs (Farrell, Pappalardo, Shelanski, 2010).

${ }^{13}$ From (5), note that $\frac{d}{d t} E[q \mid q<t]<1$ if and only if $\int_{0}^{t} F(q) d q f(t)<F(t) F(t)$, which is implied by the logconcavity of $\int_{0}^{t} F(q) d q$ as seen from the second derivative of $\ln \left(\int_{0}^{t} F(q) d q\right)$. Logconcavity is inherited by integration so logconcavity of $f$ implies logconcavity of $F$ and hence of $\int_{0}^{t} F(q) d q$, so this proves the second part of Conditional Means Property (ii). The first part of this standard property can be shown analogously. 
and the second derivative evaluated at any point where $\frac{d}{d t} E[q \mid q<t]=1 / 2$ is then

$$
\left[\frac{d^{2} E[q \mid q<t]}{(d t)^{2}}\right]_{\frac{d}{d t} E[q \mid q<t]=1 / 2}=\frac{\int_{0}^{t} F(q) d q f^{\prime}(t)}{F(t) F(t)}<0 .
$$

Therefore if $\frac{d}{d t} E[q \mid q<t]=1 / 2$ for any $t=t^{\prime}$ then $\frac{d}{d t} E[q \mid q<t]<1 / 2$ for all $t>t^{\prime}$. Considering the limit as $t$ goes to 0 , the truncated distribution approximates a uniform distribution, so the derivative should converge to $1 / 2$. Checking, we apply l'Hôpital's rule twice to find $\lim _{t \rightarrow 0} \frac{d}{d t} E[q \mid q<t]=1 / 2$. Therefore $\frac{d}{d t} E[q \mid q<t]<1 / 2$ for all $t>0$. From (4),

$$
\frac{d(E[q \mid q>t])}{d t}=\frac{d}{d t}\left(\frac{1-t F(t)}{1-F(t)}-\frac{\int_{t}^{1} F(q) d q}{1-F(t)}\right)=\frac{f(t)\left(1-t-\int_{t}^{1} F(q) d q\right)}{(1-F(t))^{2}}
$$

and the second derivative evaluated at any $t$ where $\frac{d}{d t} E[q \mid q>t]=1 / 2$ is

$$
\left[\frac{d^{2} E[q \mid q>t]}{(d t)^{2}}\right]_{\frac{d(E[q \mid q>t])}{d t}=1 / 2}=\frac{(1-F(t))^{2}\left(1-t+\int_{t}^{1} F(q) d q\right) f^{\prime}(t)}{(1-F(t))^{4}}<0
$$

so if $\frac{d}{d t} E[q \mid q>t]=1 / 2$ for any $t=t^{\prime}$ then $\frac{d}{d t} E[q \mid q>t]>1 / 2$ for any $t<t^{\prime}$. Considering the limit as $t$ goes to 1 , there are two cases. The first case is when $f(1)>0$, in which case the truncated distribution approximates a uniform distribution. Checking, we apply l'Hôpital's rule twice to find $\lim _{t \rightarrow 1} \frac{d}{d t} E[q \mid q>t]=1 / 2$ so for this case $\frac{d}{d t} E[q \mid q>t]>1 / 2$ for $t<1$. The second case is when $f(1)=0$, in which case the truncated distribution approximates a triangle distribution, so the derivative should go to $2 / 3$. Checking, we apply l'Hôpital's rule a third and fourth time to find $\lim _{t \rightarrow 1} \frac{d}{d t} E[q \mid q>t]=2 / 3$. Therefore for this case $\frac{d}{d t} E[q \mid q>t]>1 / 2$ for all $t$ because otherwise the first derivative must cross $1 / 2$ from below which is inconsistent with the above result that the derivative is falling when the derivative is $1 / 2$.

(v) If $f^{\prime}>0$, the same calculations imply $\frac{d}{d t} E[q \mid q<t] \geq \frac{1}{2}, \frac{d}{d t} E[q \mid q>t] \leq \frac{1}{2}$.

Proposition 5 For any quality density $f$, the most informative feasible grading scheme is either pass-fail or honors.

PROOF: (1) We first show that the most informative grading scheme is either pass-fail, honors, or exact grading. Consider an alternative scheme with $a$ as the lowest type to be certified and $b$ as the lowest type to be exactly revealed. For this scheme to be different from one of the three schemes and superior to them it must be feasible and either: 
(i) A positive measure of types in $[a, b)$ are not completely pooled together, and/or

(ii) A positive measure of types in $[b, 1]$ are not exactly revealed.

Regarding case (i), if a positive measure of types in $[a, b)$ are not completely pooled, consider any two positive measure subsets of non-pooling types $A, B \in[a, b)$. If $E[q \mid q \in$ $A] \neq E[q \mid q \in B]$ then there is a positive measure of types in either $A$ or $B$ who can be feasibly exactly revealed without affecting the feasibility of certification for other types in the subset, thereby lowering MSE.

If instead $E[q \mid q \in A]=E[q \mid q \in B]$, the total MSE for these types is

$$
\begin{aligned}
\int_{q \in A}(q-E[q \mid q & \in A])^{2} f(q) d q+\int_{q \in B}(q-E[q \mid q \in B])^{2} f(q) d q \\
& =\int_{q \in A \cup B} q^{2} f(q) d q-E[q \mid q \in A]^{2} \int_{q \in A \cup B} f(q) d q
\end{aligned}
$$

which is the same as if all types in $A$ and $B$ pool, so there is no gain in informativeness from not pooling.

Regarding case (ii), suppose a positive measure of types in $[b, 1]$ are not exactly revealed. If certification is feasible for types in $[a, b]$ it is also feasible for all types in $[b, 1]$, so MSE falls if any positive measure of types in $[b, 1]$ that are not exactly revealed are instead exactly revealed.

(2) Given that the most informative scheme is one of the three schemes, our next task is to show that the best scheme is either pass-fail or honors.

Consider the lowest feasible exact scheme $x^{*}$ where all types $q \geq x^{*}$ apply for certification, i.e., $x^{*}$ is the lowest $x$ such that $x-E[q \mid q<x]=c$. This scheme is the most informative exact scheme. First consider an honors scheme $(p, x)$ where $x=x^{*}$. Define the function $p(x)$ as the lowest $p$ feasible for $x$ and the given $c$ :

$$
p(x) \equiv \min \{p: E[q \mid q \in[p, x)]-E[q \mid q \in[0, p)]=c\} .
$$

Note that $p(x) \leq x$ for feasible $x$ since if $x$ is feasible then at least the one-type pool $[x, x]$ is feasible. If $p\left(x^{*}\right)<x^{*}$ then the honors scheme $\left(p\left(x^{*}\right), x^{*}\right)$ is more informative than the exact scheme $x^{*}$ and honors grading is superior to exact grading (though pass-fail might be even better; see Proposition 2).

So suppose instead that $p\left(x^{*}\right)=x^{*}$, which means that a feasible honors scheme requires $x>x^{*}$. Since $E[q \mid q \in[p, x)]-E[q \mid q \in[0, p)]$ is continuous in $p$ and $x$ (recalling 
that $f(q)$ is assumed to be atomless), the function $p(x)$ is continuous in the neighborhood below $p\left(x^{*}\right)=x^{*}$. Since $E[q \mid q \in[p, x]]-E[q \mid q \in[0, p)]$ is strictly increasing in $x$, the derivative of $p(x)$ is negative: $\frac{d p}{d x}<0$. Therefore starting at $x=x^{*}$ so that $p\left(x^{*}\right)=x^{*}$, we raise $x$ above $x^{*}$ and $p(x)$ falls. Note that if an honors scheme is feasible for types in the pass region it must be feasible for higher types in the exact region, so such a change is feasible. We are interested in whether the mean squared error,

$$
\int_{0}^{p(x)}(q-E[q \mid q<p(x)])^{2} f(q) d q+\int_{p(x)}^{x}(q-E[q \mid q \in[p(x), x]])^{2} f(q) d q,
$$

is decreasing in $x$, thereby implying that $M S E_{\text {honors }}<M S E_{\text {exact }}$. The derivative of (11) with respect to $x$ is

$$
\begin{aligned}
& \frac{d p}{d x}(p(x)-E[q \mid q<p(x)])^{2} f(p(x)) \\
& -2\left(\frac{d}{d x} E[q \mid q<p(x)]\right) \int_{0}^{p(x)}(q-E[q \mid q<p(x)]) f(q) d q \\
& +(x-E[q \mid q \in[p(x), x]])^{2} f(x) \\
& -\frac{d p}{d x}(p(x)-E[q \mid q \in[p(x), x]])^{2} f(p(x)) \\
& -2\left(\frac{d}{d x} E[q \mid q \in[p(x), x]]\right) \int_{p(x)}^{x}(q-E[q \mid q \in[p(x), x]]) f(q) d q .
\end{aligned}
$$

The third and fifth terms are zero when $p(x)=x^{*}=x$, the particular case we are looking at. The fourth term equals zero because $E[q \mid q \in[p(x), x]]=p(x)$ when $p=x$. The second term is zero because the integral of $q f(q)$ from 0 to $p(x)$ equals $E[q \mid q<p(x)]$. That leaves the first term, which is negative because $\frac{d p}{d x}<0$.

As a result, starting from the exact scheme $p=x=x^{*}$ the mean squared error can always be reduced by increasing $x$ and creating an honors scheme, so $M S E_{\text {honors }}<$ $M S E_{\text {exact }}$.

Proposition 6 i) For c sufficiently low and any quality density $f$, honors grading is most informative, exact grading is next, and pass-fail is least informative:

$$
M S E_{\text {honors }}<M S E_{\text {exact }}<M S E_{\text {pass-fail }} .
$$

ii) For c sufficiently high and $f$ decreasing, if any scheme is feasible then pass-fail grading is most informative, honors is next, and exact grading is least informative:

$$
M S E_{\text {pass-fail }}<M S E_{\text {honors }}<M S E_{\text {exact }} .
$$


PROOF: (i) We know from the proof of Proposition 1 that $M S E_{\text {honors }}<M S E_{\text {exact }}$ for any $c$ such that either scheme is feasible, which is the case for $c$ sufficiently small. For sufficiently small $c$ it must be that $M S E_{\text {exact }}<M S E_{\text {pass-fail }}$ because as $c$ approaches zero $M S E_{\text {exact }}$ approaches 0 , while $M S E_{\text {pass-fail }}$ is always bounded from below by either the pass pool or the fail pool's contribution to MSE:

$$
\min _{p} \max \left\{\int_{0}^{p}(q-E[q \mid q<p])^{2} f(q) d q, \int_{p}^{1}(q-E[q \mid q \geq p])^{2} f(q) d q\right\}>0 .
$$

Since honors grading is better than exact grading, it is also better than pass-fail grading for low $c$.

(ii) We want to show that $M S E_{\text {pass-fail }}<M S E_{\text {honors }}$ for $c$ sufficiently high if $f$ is decreasing in quality and some scheme remains feasible. Since $f$ is decreasing, $F$ is concave, and its concave transformation $\ln (F)$ is also concave, i.e., $F$ is log-concave. Therefore, for any value $x, \frac{d}{d x} E[q \mid q<x]<1$ by Conditional Means Property (ii) and hence $\frac{d}{d x}(x-E[q \mid q<x])>0$ so the best exact scheme $x^{*}$ is increasing in $c$ and approaches 1 as $c$ approaches $1-E[q]$. Property (iv) tells us that since $f$ is decreasing, the gap $E[q \mid q \in[p, x)]-E[q \mid q<p]$ is increasing in $p$. If $x=x^{*}$, so type $x$ is barely willing to be exactly revealed, then the lowest feasible $p$ is also $x^{*}$, or, using again the function $p(x)$ defined in Proposition 1, $p\left(x^{*}\right)=x^{*}$. Therefore, as established in that proposition, MSE falls as $x$ rises from $x^{*}$ and $p(x)$ decreases with it. This implies that for $c$ high enough that $x^{*}$ is close to 1 , MSE is higher for the scheme with $x=1$ and $p=p(1)<1$ than for the exact scheme $x^{*}$. But such a scheme is in fact not an honors scheme, but a pass-fail scheme with $p^{*}=p(1)$. Can an honors scheme with $x<1$ do better? Such a scheme must have $x>x^{*}$ to be feasible, but for $x$ close to $x^{*}$ MSE is strictly decreasing, so for $c$ high enough that $x^{*}$ is sufficiently close to 1 the best scheme is $p^{*}=p(1)$.

Proposition 7 (i) For any quality density $f$, if exact grading is feasible then so is honors grading, and if honors grading is feasible then so is pass-fail. (ii) For $f$ increasing, or for $f$ log-concave and $E[q]>1 / 2$, pass-fail and honors grading are feasible for a range of grading costs so high that exact grading is not.

PROOF: (i) Recall that pass-fail grading supports an equilibrium if $c \leq E[q \mid q \geq p]-$ $E[q \mid q<p]$ for some $p<1$, and exact grading supports an equilibrium if $c \leq x-E[q \mid q<x]$ for some $x<1$. Since $E[q \mid q \geq p]>p$ for $p<1$, it must be that

$$
\max _{p}\{E[q \mid q \geq p]-E[q \mid q<p]\}>\max _{x}\{x-E[q \mid q<x]\}
$$


unless $p=x=1$, in which case no firms apply (or, if the reader prefers, just the infinitesimal amount $x=1$ ). Moreover, since inequality (13) is strict, for given exact grading participation cutoff $x^{*}$, pass-fail would be still be feasible even if $p$ were picked not optimally, but slightly too small, at $p^{\prime}$. For honors grading, pick $p=p^{\prime}$ and pick $x$ such that $p(x)=p^{\prime}$, where $p(x)$ is the function defined in equation (10). By the continuity of $p(x)$ proved earlier, if $p^{\prime}$ is chosen close enough to $p$ (but $\left.p^{\prime}<p\right),\left(p^{\prime}, x\right)$ will then be a feasible honors scheme.

(ii) For the first condition, note that if $f$ is increasing then by Conditional Means Property (v) the pass-fail scheme that is feasible for the highest $c$ has $p=0$ and is feasible for $c \leq E[q \mid q \geq 0]-0=E[q]$. Similarly an honors scheme where only type $q=0$ fails and only type $q=1$ is exactly revealed also exists under this same condition. So if $\max _{x}\{x-E[q \mid q<x]\} \leq 1 / 2$ exact grading is not feasible when passfail grading and honors grading are. Since $f$ is increasing $E[q \mid q<x]>x / 2$, so $\max _{x}\{x-E[q \mid q<x]\}<\max _{x}\{x-x / 2\}=1 / 2$. For the second condition, note that for $f$ log-concave, $\arg \max _{x}\{x-E[q \mid q<x]\}=1$ by Conditional Means Property (ii) and exact grading is feasible for $c<1-E[q]$. Since a pass-fail scheme where only type $q=0$ fails and a corresponding honors grading scheme with $p=0$ and $x=1$ are both

supported for $c<E[q]$, the condition for exact grading is tighter than for pass-fail and honors if $1-E[q]<E[q]$ or $E[q]>1 / 2$.

\section{References}

[1] BAGNOLI, MARK \& TED BERGSTROM (2005): "Log-Concave Probability and Its Applications," Economic Theory, 26, 445-469.

[2] BENABOU, ROLAND \& JEAN TIROLE (2005): "Incentives and Prosocial Behavior," American Economic Review, 96, 1652-1678

[3] CHAKRABORTY, ARCHISHMAN \& RICK HARBAUGH (2007): "Comparative Cheap Talk," Journal of Economic Theory, 132, 70-94.

[4] CHAN, WILLIAM, LI HAO \& WING SUEN (2007): "A Signaling Theory of Grade Inflation," International Economic Review, 48, 1065-1090.

[5] COSTRELL, ROBERT M. (1994): "A Simple Model of Educational Standards," American Economic Review, 84, 956-971. 
[6] CRAWFORD,VINCENT P. \& JOEL SOBEL (1982) "Strategic Information Transmission," Econometrica, 50, 1431-1451.

[7] DUBEY, PRADEEP \& JOHN GEANAKOPLOS (2006): "Grading in Games of Status: Marking Exams and Setting Wages," working paper, CFDP 1544R.

[8] FARHI, EMMANUEL, JOSH LERNER, \& JEAN TIROLE (2011): "Fear of Rejection? Tiered Certification and Transparency," NBER Working Paper No. 14457.

[9] FARRELL, JOSEPH, JANIS K. PAPPALARDO, AND HOWARD SHELANSKI (2010): "Economics at the FTC: Mergers, Dominant-Firm Conduct, and Consumer Behavior," Review of Industrial Organization, 37, 263-277.

[10] JEWITT, IAN (2004): "Notes on the Shape of Distributions," working paper, Nuffield College, Oxford.

[11] KAMENICA, EMIR \& MATTHEW GENTZKOW (2011): "Bayesian Persuasion," American Economic Review, 101, 2590-2615.

[12] LIZZERI, ALESSANDRO (1999): "Information Revelation and Certification Intermediaries," RAND Journal of Economics, 30, 214-231.

[13] MOLDOVANU, BENNY, ANER SELA \& XIANWEN SHI (2007): "Contests for Status," Journal of Political Economy, 115, 338-363.

[14] MORGAN, JOHN \& PHILLIP C. STOCKEN (2003): “An Analysis of Stock Recommendations," RAND Journal of Economics, 34, 183-203.

[15] OSTROVSKY, MICHAEL \& MICHAEL SCHWARZ (2010): "Information Disclosure and Unraveling in Matching Markets," American Economic Journal: Microeconomics, 2, 34-63.

[16] RAYO, LUIS \& ILYA SEGAL (2010): "Optimal Information Disclosure," Journal of Political Economy, 118, 949-987.

[17] TITMAN, SHERIDAN \& BRETT TRUEMAN (1986): "Information Quality and the Valuation of New Issues," Journal of Accounting and Economics, 8, 159-172. 